\title{
Strings and Super-Yang-Mills theory: the integrable story
}

\author{
Sakura Schäfer-Nameki \\ California Institute of Technology, 1200 E California Boulevard, Pasadena, \\ CA 91125, USA \\ E-mail: ss299@theory.caltech.edu
}

Received 8 November 2006

Accepted 10 November 2006

Published 19 December 2006

Online at stacks.iop.org/JSTAT/2006/N12001

doi:10.1088/1742-5468/2006/12/N12001

The most prominent role that string theory has played in recent years, both in science and in the media, has been that of a candidate theory of everything. What is often forgotten in heated discussions on the actual relevance of string theory for real world physics is the productive and vital influence that string theoretical considerations have upon mathematics, ranging all the way from geometry and topology to algebra. Key to this dialogue between mathematics and string theory is not only the successful application of fancy mathematics to ten-dimensional string theories, but also the substantial inflow of creative, unthought-of mathematical ideas and problems concerning e.g. Calabi-Yau manifolds, topological invariants and Kac-Moody algebras that naturally arise in the context of string theory.

Recently, a similarly enticing connection between string theory and integrable systems has emerged, the focus of interest being on integrable spin chains with Lie superalgebra symmetries and long-range interactions. Most of the questions that are being addressed at present are tailored to the particular problem that is posed within string theory; however a lot of mileage has come out of the research with respect to general aspects of long-range interacting quantum spin chains. A particularly interesting paper in this respect is the work by Beisert and Klose [1]. Before elaborating on the results in this paper, we should set the scene and recap some of the observations that led to the present raised awareness of integrability within string theory.

The AdS/CFT conjecture in string theory predicts that the strong-coupling limit of a certain supersymmetric four-dimensional, superconformal Yang-Mills gauge theory, $\mathcal{N}=$ $4 S U(N)$ SYM, is described by perturbative string theory on a five-dimensional hyperbolic space, times a five-sphere, $\mathrm{AdS}_{5} \times S^{5}$. In terms of the spectrum the correspondence 
predicts agreement of the eigenvalues $\Delta$ of the dilatation generator $\mathcal{D}$ on gauge-invariant operators of the SYM theory with the energies $E$ of quantum strings in $\operatorname{AdS}_{5} \times S^{5}$. Similar conjectures have been put forward for less supersymmetric or non-supersymmetric cousins of $\mathcal{N}=4 \mathrm{SYM}$ and validity of the proposed dual string description would indeed teach us a lot about QCD at strong coupling. This conjecture has to date been difficult to prove, because the perturbative regimes of the two theories are disjoint: the SYM theory is perturbative around $\lambda=0$, where $\lambda$ is the ('t Hooft) coupling constant, whereas the string theory is perturbative in $1 / \sqrt{\lambda}$. Moreover, the direct perturbative computations of $\Delta(\lambda)$ and $E(1 / \sqrt{\lambda})$ are cumbersome and limited to a few perturbative orders, so we are in dire need of an improved conceptual understanding of the duality. The ultimate aim is to derive an exact function $f(\lambda)$, which when expanded around small values of $\lambda$ yields the SYM scaling dimensions $\Delta(\lambda)$, and likewise for large $\lambda$ reproduces the string energies $E(1 / \sqrt{\lambda})$.

Major progress towards this end has been achieved following the insight of Minahan and Zarembo [2], that the spectrum of anomalous dimensions in planar (meaning, $N$ is infinite) $\mathcal{N}=4 \mathrm{SYM}$ at one-loop level is the same as the spectrum of a nearestneighbour Heisenberg $X X X$ ferromagnetic spin chain. To illustrate this, consider the subsector of the SYM theory containing only two complex scalar fields $\mathcal{Z}$ and $\mathcal{W}$. The generic gauge-invariant operator in this sector is a trace with insertions of scalars: $\mathcal{O}=\operatorname{Tr}(\mathcal{Z} \mathcal{Z} \mathcal{W} \mathcal{W} \mathcal{W} \cdots)+$ permutations. Identifying the scalars with the two states in the $\mathfrak{s u}(2)$ spin $1 / 2$ representation, $\mathcal{Z}=|\uparrow\rangle$ and $\mathcal{W}=|\downarrow\rangle$, and opening the trace

$$
\operatorname{Tr}(\mathcal{Z Z W Z W W} \cdots) \rightarrow|\uparrow \uparrow \downarrow \uparrow \downarrow \downarrow \cdots\rangle,
$$

it was shown in [2] that the one-loop dilatation operator $\mathcal{D}_{1}$ is precisely acting on the resulting spin chain as the Heisenberg $X X X$ Hamiltonian:

$$
\mathcal{D}_{1} \rightarrow H_{X X X}=\sum_{i=1}^{L}\left(1-P_{i, i+1}\right) .
$$

The ground state is the operator of lowest anomalous dimension, which is the ferromagnetic state $\operatorname{Tr}\left(\mathcal{Z}^{L}\right)$ and periodicity is imposed. Thus, finding the eigenvalues $\Delta_{1}$ of $\mathcal{D}_{1}$ is equivalent to solving for the Heisenberg $X X X$ ferromagnet, which can be accomplished by means of the Bethe ansatz. This simple picture extends to all operators of $\mathcal{N}=4 \mathrm{SYM}$ at one-loop level, where the anomalous dimensions can be obtained from a Heisenberg, nearest-neighbour spin chain with superalgebra symmetry $\mathfrak{p s u}(2,2 \mid 4)$. Furthermore, this insight was complemented by the, albeit classical, integrability of the dual string theory on $\mathrm{AdS}_{5} \times S^{5}$. Finite-gap solutions to the Bethe equations for SYM in the thermodynamic limit ${ }^{1}$ can be mapped (in a certain parameter regime) to finite-gap solutions of the classical string theory [3].

Integrability of the SYM theory extends to higher loops, where the associated spin chains are long-range interacting, with the interaction range growing linearly with the order in perturbation theory in $\lambda$ (nearest neighbours at one-loop level, (next-to $)^{p-1}$ nearest neighbours at $p$-loop level), and the standard dispersion relation is replaced by

\footnotetext{
1 This limit differs from the thermodynamic limit in the integrable literature, in that $L \rightarrow \infty$, while the filling fractions are kept finite.
} 
the $\lambda$-dependent relation

$$
\epsilon(p)=\sqrt{1+\frac{\lambda}{\pi^{2}} \sin ^{2}\left(\frac{p}{2}\right)} .
$$

Despite similarities with relativistic and lattice dispersion relations, long-range spin chain models with this property have so far not appeared in the literature. The construction of Bethe ansätze for these models is further obstructed as the spin chain Hamiltonian, i.e. the dilatation operator of $\mathcal{N}=4 \mathrm{SYM}$, is only known perturbatively up to a certain number of loops. However, in a remarkable paper [4] Beisert has recently constructed an $S$-matrix for general long-range interacting, integrable quantum spin chains with centrally extended $\mathfrak{s u}(2 \mid 2)^{2}$ symmetry, which in particular fixes the $S$-matrix of the SYM theory up to a scalar dressing factor. The construction solely relies on the symmetries of the problem, and the $S$-matrix is proven to satisfy the Yang-Baxter equations. Here, it may be of interest to mention that this $S$-matrix is equivalent to Shastry's $R$-matrix of the Hubbard model, which thereby has a so-far unknown hidden supersymmetry [4].

In view of these curious integrable structures in the SYM theory, it is then natural to propose a Bethe ansatz to describe the dual string theory on $\operatorname{AdS}_{5} \times S^{5}$ beyond the classical discussion of [3]. If the AdS/CFT correspondence is to be correct, this should in a certain regime coincide with the Bethe ansätze for $\mathcal{N}=4 \mathrm{SYM}$. The spin chain picture is a lot less clear on the string theory side; however, the string Bethe ansatz seems to reproduce all the known data for energies of infinitely long strings. Since the string theory shares the same symmetry algebra as the SYM theory, the construction of the $S$-matrix by Beisert should apply likewise. Thus the only difference between the SYM and string $S$-matrices is in the choice of the scalar dressing factor. Most remarkably it was shown recently that a phase which interpolates correctly between strong and weak coupling seems to exist [5]. This is very exciting and seems to imply that further study of the integrable structure of AdS/CFT may in fact at some point lead a proof of the correspondence in the planar limit!

Following these developments, various detailed studies of spin chains with long-range interactions, and non-standard (non-relativistic) dispersion relations, which do not seem to have been discussed elsewhere in the literature, as well as the associated asymptotic Bethe ansätze, have appeared. The chains are generically massive, and have periodic boundary conditions, due to the trace that appears in the SYM operators. The paper by Beisert and Klose [1] is placed in this context. The core of the paper is an analysis of the most general long-range $\mathfrak{g l}(n)$ spin chains with matter in the fundamental representation. This in itself is not directly relevant for the spin chain problems that $\mathcal{N}=4 \mathrm{SYM}$ poses, but has several common features: the interaction is not nearest neighbour and the model is solved perturbatively by means of an asymptotic, nested Bethe ansatz. Asymptoticity states that the range of interactions remains smaller than the total length of the spin chain.

The construction of the general $\mathfrak{g l}(n)$ spin chain relies on a perturbative analysis in a parameter $\lambda$, which governs the range of interaction, and which in the $\mathcal{N}=4$ SYM spin chain is provided by the coupling constant. The assumption that goes into the construction of Beisert and Klose is that it is sufficient to consider two commuting charges: $\mathcal{Q}_{2}(\lambda)$, which is the Hamiltonian, and an additional higher charge $\mathcal{Q}_{3}(\lambda)$, and to ensure that these commute at each perturbative order in $\lambda$. The construction of the most general 
such charges is performed up to fourth order in perturbation theory. Furthermore, an all-loop asymptotic Bethe ansatz is proposed.

Perhaps most interestingly in view of the AdS/CFT correspondence are two points made in the paper: the general all-loop ansatz has motivated generalizations of the proposed Bethe ansätze, e.g. the ansatz for the generalized scalar phase $\theta$ has entered the dressing factors for the $S$-matrices of SYM and strings and is crucial to the interpolation between strong and weak coupling in [5]. Secondly, as a corollary to the classification of all $\mathfrak{g l}(n)$ spin chains, it is shown that the plane-wave matrix model, which is closely related to $\mathcal{N}=4 \mathrm{SYM}$, is not fully integrable. Like $\mathcal{N}=4 \mathrm{SYM}$ it has subsectors in which integrability is shown to a certain loop order. However, this model is not of the general integrable type found in [1] and thus, is not all-loop integrable. This result does not have implications for the integrability of the $\mathcal{N}=4$ spin chain; however it teaches a basic lesson, that perturbative integrability can in fact break down at a certain loop order.

String theorists have in recent years learned much about integrable models by profiting from decades of research into quantum spin chains, Bethe ansätze and classical integrable systems. The integrable models which have emerged from AdS/CFT and methods for analysing them, however, seem to go beyond the known and well-explored terrain, hopefully this will spark the interest of experts in the statistical mechanics community to further explore the structure of these models.

\section{References}

[1] Beisert N and Klose T, Long-range gl(n) integrable spin chains and plane-wave matrix theory, 2006 J. Stat. Mech. P07006 [hep-th/0510124]

[2] Minahan J A and Zarembo K, The Bethe-ansatz for $N=4$ super Yang-Mills, 2003 J. High Energy Phys. JHEP03(2003)013 [hep-th/0212208]

[3] Kazakov V A, Marshakov A, Minahan J A and Zarembo K, Classical/quantum integrability in AdS/CFT, 2004 J. High Energy Phys. JHEP05(2004)024 [hep-th/0402207]

[4] Beisert N, The analytic Bethe ansatz for a chain with centrally extended su(2|2) symmetry, 2006 Preprint nlin.SI/0610017

[5] Beisert N, Eden B and Staudacher M, Transcendentality and crossing, 2006 Preprint hep-th/0610251 